\title{
Distinctions of dendritic behavior influenced by constant pressure and periodic pressure
}

\author{
Shan Shang ${ }^{1,2}$, Zhi-peng Guo ${ }^{3}$, Zhi-qiang Han ${ }^{4}$, 'Xin-yu Zhang ${ }^{1,2}$, **Yi-nuo Cheng ${ }^{5}$, Jun Li' ${ }^{1,2}$ \\ 1. State Key Laboratory of Automotive Safety and Energy, Tsinghua University, Beijing 100084, China \\ 2. School of Vehicle and Mobility, Tsinghua University, Beijing 100084, China \\ 3. Beijing Supreium Co., Ltd., Beijing 100089, China \\ 4. School of Materials Science and Engineering, Tsinghua University, Beijing 100084, China \\ 5. Beijing Jingwei Hirain Technologies Co., Inc., Beijing 100191, China
}

\begin{abstract}
The distinctions of dendritic morphology and sidebranching behavior when solidified under atmosphere pressure, constant pressure which is higher than atmosphere pressure (hereinafter referred to as constant pressure) and periodic pressure were investigated using 3-D phase field method. When growing at atmosphere pressure, side branches (secondary dendritic arms) are irregular. When solidified under constant pressure with a relatively high value, side branches are much more luxuriant, with more developed high-order side branches. When applied with periodic pressure, resonant sidebranching happens, leading to many more regular side branches and the smallest secondary dendritic arm spacing (SDAS) in the three cases. The significant difference in dendritic morphology is associated with tip velocity modulated by total undercooling including pressure and temperature undercooling. In the case of constant pressure, tip velocity increases linearly with total undercooling, and it varies periodically in periodic pressure case. The different variation trend in tip velocity is the reason for the distinct dendrite growth behavior in different cases. Unlike the phenomenon in constant pressure case where the dendrite grows faster with higher pressure, the dendrite grows slower under periodic pressure with higher amplitude, resulting in less developed primary dendrite and side branches. This is influenced by tip remelting due to low undercooling or even negative undercooling. It is revealed that the accelerated velocity of tip remelting increases with the decline of undercooling. The greater the amplitude of periodic pressure, the faster the tip remelting velocity during one period. This is the reason why the average tip velocity decreases with the rise of amplitude of periodic pressure.
\end{abstract}

Key words: periodic pressure; sidebranching; amplitude; tip velocity; undercooling; phase field method
CLC numbers: TG146.21/TP391.9
Document code: A
Article ID: 1672-6421(2021)02-094-07

\section{Introduction}

Dendrite morphology during solidification has a significant influence on mechanical properties. The feature of side branches, especially secondary dendritic arm spacing (SDAS), is associated with strength and toughness of alloys. It is of great importance to investigate the effect of external perturbation, such as heat and pressure, on dendritic growth and sidebranching ${ }^{[1-10]}$. According to the

\section{*Xin-yu Zhang}

Male, Master, Senior Engineer. His research interests mainly focus on the intelligent vehicles and robotics, machine learning, deep learning, and driving safety.

E-mail: xyzhang@tsinghua.edu.cn;

**Yi-nuo Cheng

E-mail: yinuo.cheng@hirain.com;

Received: 2020-11-22; Accepted: 2021-01-07 study by LaCombe et al. ${ }^{[4-6]}$, pressure altered the state of dendrite growth due to the change of melting points, and determined sidebranching behavior. Therefore, pressure is a nonnegligible factor in determining dendritic growth and sidebranching, and has been investigated experimentally and numerically ${ }^{[11-13]}$.

When it comes to a numerical method, the phase field method ${ }^{[14-18]}$ which takes thermal dynamics under pressure into consideration, has become a powerful tool to study dendritic growth and sidebranching during pressurized solidification ${ }^{[19-23]}$. According to phase field research, when applied with constant pressure, the growth of dendrites is promoted, with raised tip velocity and developed dendritic arms ${ }^{[19]}$. When applied with periodic pressure, dendrite morphology and sidebranching frequency can be changed dramatically, since tip velocity is modulated by the periodic pressure ${ }^{[22,23]}$. The underlying mechanism for 
dendritic sidebranching has been explored by applying periodic pressures at different frequencies by using the 3-D phase field modeling method ${ }^{[20]}$. It is revealed that if the frequency of applied pressure is in an appropriate range, both tip velocity and sidebranching would be completely synchronized with the pressure, resulting in regular side branches with high sidebranching frequency.

Even though the influence of constant pressure and periodic pressure on dendrite morphology has been studied separately, distinctions between dendrite growth behavior under the two kinds of pressures and the underlying mechanism have not been explored yet.

In this work, dendritic morphology and sidebranching behavior under atmosphere pressure, constant pressure and periodic pressure were investigated using the 3-D phase field method. The features and distinctions of dendrite morphology and side branches in the three cases were demonstrated. The undercooling and tip velocity modulated by different pressures were compared to reveal the mechanism of the difference in sidebranching behavior. The great influence of the amplitude of periodic pressure on sidebranching behavior was explored by investigating the correlation between tip remelting velocity with low undercooling and even negative undercooling.

\section{Mathematical model}

The 3-D phase field model has been described in Ref. [20] in detail. The 3-D controlling equations (anisotropic) for phase field and solute field ${ }^{[16,17,24]}$ are as follows:

$$
\begin{gathered}
\tau \frac{\partial \phi}{\partial t}=\nabla \cdot\left(W(\vec{n})^{2} \nabla \phi\right)+\frac{\partial}{\partial x}\left(|\nabla \phi|^{2} W(\vec{n}) \frac{\partial W(\vec{n})}{\partial \phi x}\right) \\
+\frac{\partial}{\partial y}\left(|\nabla \phi|^{2} W(\vec{n}) \frac{\partial W(\vec{n})}{\partial \phi y}\right)+\frac{\partial}{\partial z}\left(|\nabla \phi|^{2} W(\vec{n}) \frac{\partial W(\vec{n})}{\partial \phi_{z}}\right) \\
+\phi\left(1-\phi^{2}\right)+\lambda\left(1-\phi^{2}\right)^{2}(\theta+k U) \\
\left(\frac{1+k}{2}-\frac{1-k}{2}\right) \frac{\partial u}{\partial t}=\nabla \cdot\left(D \frac{1-\phi}{2} \nabla U-\vec{J} a t\right)+\frac{1}{2}[1+(1-k) U] \frac{\partial \phi}{\partial t}
\end{gathered}
$$

where $\tau$ is the relaxation time, $\phi$ is phase field which is -1 in bulk liquid and 1 in bulk solid, $W(\vec{n})$ is the anisotropic width of the diffuse interface with $\vec{n}$ the unit normal vector pointing out into the liquid, $k$ is the partition coefficient for solute, and $D$ is solute diffusivity. $\lambda$ is the scaling parameter and defined as:

$$
\lambda=\frac{15}{16} \frac{R T_{M}(1-k)}{v_{0} H|m|} \Delta T_{0}
$$

where $R$ is the gas constant, $T_{\mathrm{M}}$ is the melting temperature of the solvent, $v_{0}$ is the molar volume, $H$ is the energy barrier of the double well potential, and $m$ is the liquidus slope in the phase diagram.

$$
\Delta T_{0}=\frac{|m| c_{\infty}(1-k)}{k}
$$

is the equilibrium freezing temperature range according to $c_{\infty}$, where $c_{\infty}$ is the initial solute concentration.

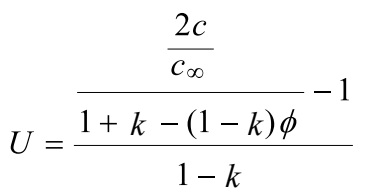

and

$$
\theta=\frac{T-T_{\mathrm{M}}-m c_{\infty}}{\Delta T_{0}}
$$

are dimensionless solute concentration and temperature, respectively, where $c$ is molar solute concentration.

$$
\vec{J} a t=-\frac{W}{\sqrt{2}} \frac{c / c_{\infty}}{[1+k-(1-k) \phi]} \frac{\partial \phi}{\partial t} \frac{\nabla \phi}{|\nabla \phi|}
$$

is the so-called "anti-trapping" current developed by Karma ${ }^{[17]}$ to counterbalance spurious effects during the phase field simulation.

In the dimensionless phase field model, the physical units of length and time are $W_{0}$ and $\tau_{0}$, respectively. Some dimensionless variables in this study, such as frequency and tip velocity, are related to the two physical units. The definitions of $W_{0}$ and $\tau_{0}$ are as follows:

$$
\begin{gathered}
W_{0}=\lambda d_{0} / a_{1} \\
\tau_{0}=d_{0}^{2} a_{2} \lambda^{3} /\left(D a_{1}^{2}\right)
\end{gathered}
$$

and

$$
d_{0}=\Gamma / \Delta T_{0}
$$

is the chemical capillary length, where $\Gamma$ is the Gibbs-Thomson coefficient which is determined by materials, $a_{1}=0.8839$ and $a_{2}=0.6267$ according to the thin interface limit analysis ${ }^{[24]}$.

$(\theta+k U)$ is the driving source term, which is related to the total undercooling of a system. Applied pressure can cause a change in remelting point and thus change the undercooling of a system. Therefore, to consider the influence of pressure, a pressure undercooling, $\xi$, caused by pressure according to the classical Clausius-Clapeyron effect was added into the source term $(\theta+k U)$ in the governing equations for the phase field. In this study, two kinds of pressure, i.e., constant pressure and periodic pressure, were applied to discover the difference of dendritic growth behavior. For constant pressure, $\xi$ is a constant value, while as to periodic pressure, $\xi$ is in the form of sinusoidal wave shown as follows:

$$
\xi=\xi_{0} \sin \left(2 \pi f_{\xi} t\right)
$$

where $f_{\xi}$ represents the frequency of pressure, $\xi_{0}$ is the amplitude of pressure undercooling. The source term acted as 
the driving force for the dendrite growth is $F_{\text {driving }}=-(\theta+k U)+\xi$, which is associated with the total undercooling caused by temperature, solute, and pressure. In this study, solute undercooling is ignored in the quantitative calculation due to the pressure has little influence on the solute undercooling and is not easy to measure, total undercooling only includes temperature undercooling and pressure undercooling.

The purpose of this study is to reveal the influence of different kinds of pressures on dendritic growth and sidebranching behavior. To focus on investigating the universal rules which apply to different alloys, the phase field model is dimensionless. The solute concentration $U$, temperature $\theta$ and pressure undercooling $\xi$ are dimensionless according to Eqs. (5) and (6), and the length and time are dimensionless based on the physical units of $W_{0}$ and $\tau_{0}$. The dimensional value of these parameters can be deduced, but they are not necessary in this study. In this study, Al-3wt.\% Cu alloy is taken as a sample alloy to simulate the dendrite growth.

In order to carry out an appropriate simulation of dendrite evolution, an initial condition was set. $\theta$ was set to be homogeneous $(\theta=-0.12)$ across the simulation domain which was about $5 \mathrm{~K}$ of undercooling for $\mathrm{Al}-3 \mathrm{wt} . \% \mathrm{Cu}$ alloy and large enough for dendrite growth. At atmosphere pressure, pressure undercooling $\xi$ was set to be zero. When studying the effect of constant pressure, pressure undercooling $\xi$ was set to be 0.05 , which represents about $2.08 \mathrm{~K}$ of undercooling for the $\mathrm{Al}-3 \mathrm{wt} . \% \mathrm{Cu}$ alloy, leading to a much greater total undercooling (about $7.08 \mathrm{~K}$ ) than that at atmosphere pressure. When investigating dendritic growth under periodic pressure, the amplitude of periodic pressure undercooling $\xi_{0}$ was set to be 0.05 as well. The frequency of pressure was set to be $0.157 / \tau_{0}$, which could cause resonant sidebranching according to Ref. [25]. Total undercooling including temperature undercooling and pressure undercooling will be extracted to study the relationship between tip velocity and undercooling quantitatively.

At the beginning of simulation, a solid seed was placed at the bottom corner of the simulation domain. A total of 30,000 time steps $(d t)$ were calculated for each simulation. Other parameters in these equations, simulation parameters, and the crystal anisotropy were set the same as those in Ref. [20]. Adaptive mesh refinement and parallel computing were adopted in this work to solve the governing equations ${ }^{[24]}$.

\section{Simulation results and discussion}

\subsection{Dendritic morphology under different kinds of pressures}

Dendrites are quadratically symmetric for Al-Cu alloy, so only $1 / 8$ dendrite is simulated to reduce simulation time. The evolution of dendritic morphology when solidified under atmosphere pressure, constant pressure, and periodic pressure is demonstrated in Fig. 1. This figure shows the dendrite

(a) $\xi=0$
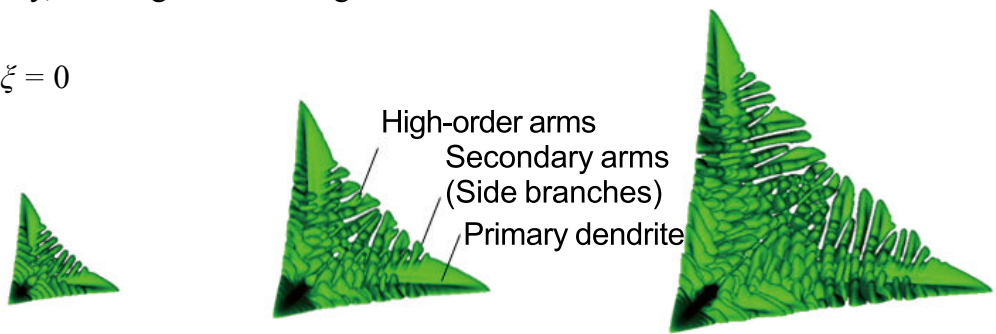

(b)

$\xi=0.05$
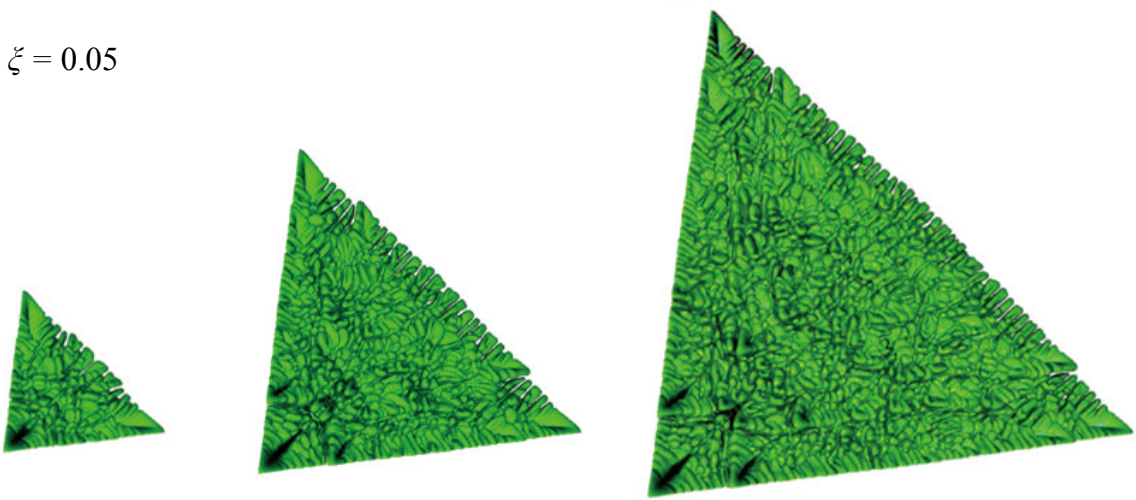

(c) $\xi=0.05 \sin \left(2 \pi f_{\xi} t\right)$
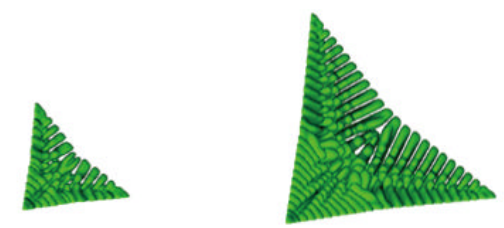

$t=10,000 d t$

$t=20,000 d t$

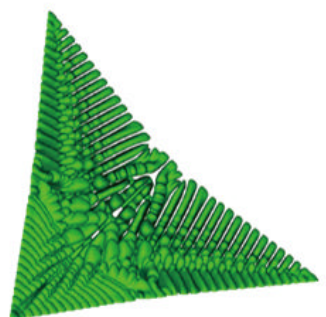

$t=30,000 d t$

Fig. 1: Dendrite morphology evolution when solidified under atmosphere pressure (a), constant pressure (b) and periodic pressure (c) 
morphology, including primary dendrite, secondary arms (side branches) and high-order arms from 10,000 dt to 30,000 dt. It is obvious that there are great distinctions in dendritic growth process in the three cases.

When growing at atmosphere pressure, side branches grow naturally. Secondary arms are relatively sparse. A dendrite tip without obvious side branches is relatively long. Highorder arms grow casually in the secondary arms. All of the sidebranching behavior results in irregular dendritic morphology.

When solidified under constant pressure which is higher than atmosphere pressure, the total undercooling including temperature undercooling and pressure undercooling is relatively high. A dendrite grows faster even at the beginning of growth $(10,000 d t)$, as shown in Fig. 1(b). The secondary arms near the root of the primary dendrite which can be termed as early secondary arms are much developed, with luxuriant high-order side branches. With the quick growth of the dendrite, those early secondary arms are larger and larger, and the high-order arms on them grow rapidly, hindering the growth of secondary arms near the dendrite tip.

Compared with those under atmosphere pressure and constant pressure, when applied with periodic pressure, side branches are much more regular [Fig.1(c)]. More secondary arms grow on the primary arms, with fewer high-order arms on them. The sidebranching frequency is the highest, that is, the SDAS is the smallest in the three cases, which may lead to the excellent physical property of alloy.

The significant difference in dendritic morphology is associated with the tip velocity which is modulated by pressure. When applied with different constant pressures, pressure undercooling varies consistently with pressure. The total undercooling for dendrite growth changes, thus affecting the dendrite growth behavior. Figure 2 shows the variation trend of tip velocity with pressure undercooling and total undercooling. As shown in Fig. 2, tip velocity increases with the value of pressure undercooling as well as total undercooling linearly. Therefore, the dendrite grows faster under higher constant pressure. When pressure is too high, side branches are crowded and wild, just like those in Fig. 1. (a)

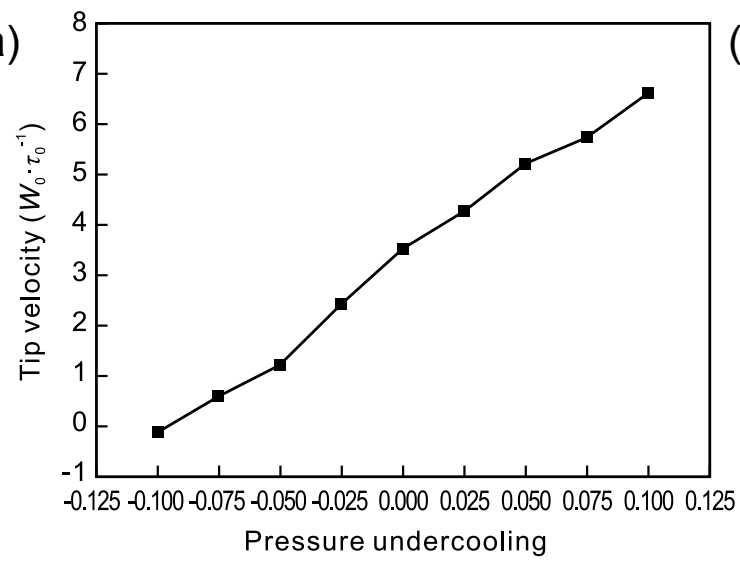

(b)

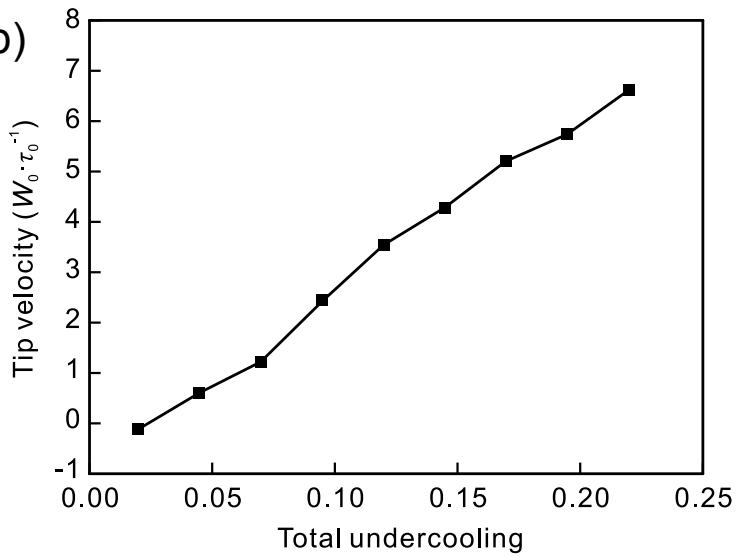

Fig. 2: Tip velocity with pressure undercooling and total undercooling under different constant pressures

To compare the modulation mechanism of different pressures, the relationship between the total undercooling and tip velocity with time in the three cases are drawn and shown in Figs. 3 and 4, respectively. When applied with

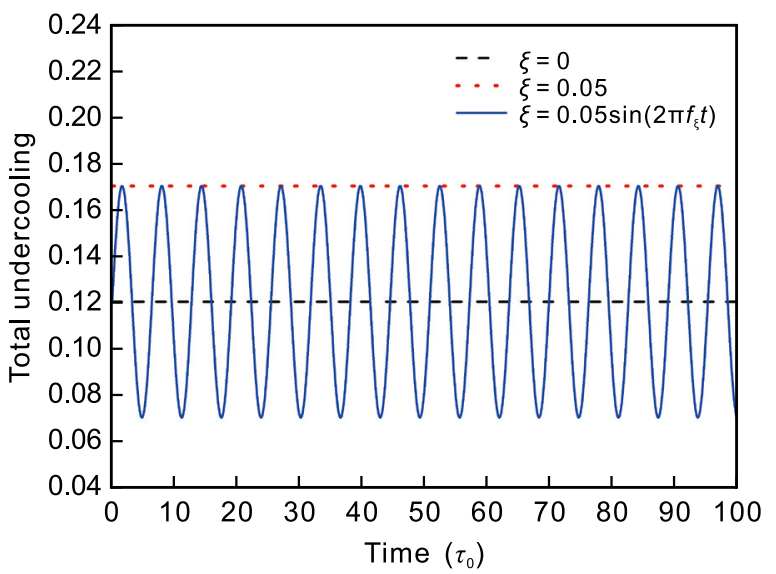

Fig. 3: Total undercooling with time under different kinds of pressures periodic pressure, total undercooling changes periodically with time. Tip velocity changes periodically and consistently with pressure and total undercooling. References [20, 25] have revealed the modulation mechanism of periodic pressure

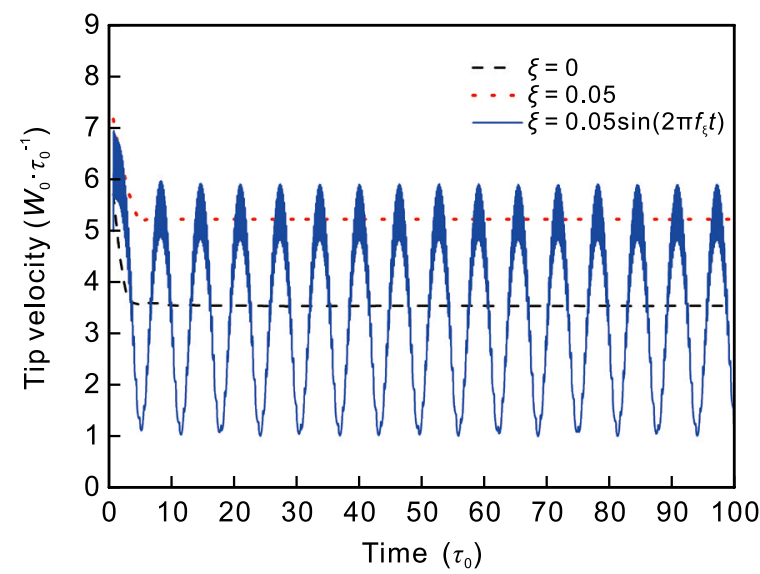

Fig. 4: Tip velocity with time under different kinds of pressures 
on dendritic sidebranching. When pressure frequency is in an appropriate range, the oscillation of tip velocity in one period results in the formation of one side branch. Resonant sidebranching occurs, and consequently, sidebranching frequency is consistent with the frequency of tip velocity and periodic pressure ${ }^{[20]}$. Resonant sidebranching under periodic pressure results in higher sidebranching frequency. This is the reason why dendrite arms are regular and SDAS is small under periodic pressure. In a word, distinctions of dendrite morphology and sidebranching behavior are determined by tip velocity which is modulated by applied pressure.

It can be seen from Fig. 3 and Fig. 4 that the curves of total undercooling are symmetrical by the value of undercooling when $\xi=0$. The curve of tip velocity when $\xi_{0}=0.05$ is also symmetrical by the value of velocity when $\xi=0$ as well.

\subsection{Dendritic morphology under periodic pressure with different amplitudes}

The amplitude of periodic pressure also executes a significant effect on dendritic growth. Note that higher $\xi_{0}$ represents higher amplitude of periodic pressure. Under the condition of fixed pressure frequency, different amplitudes $\xi_{0}$ in a range from 0.05 to 0.2 were set to investigate the influence of amplitude on dendrite morphology and sidebranching behavior. As demonstrated above, when $\xi_{0}$ is 0.05 , total undercooling is in an appropriate level for dendrite growth, resulting in regular side branches with small SDAS. If the amplitude gets higher, how dendrite morphology will evolve?

When applied with constant pressure, higher constant pressure leads to higher tip velocity due to higher pressure undercooling, as expressed by Fig. 2. Unlike the phenomenon in constant pressure case, higher amplitude does not bring about more developed dendrites and side branches. On the contrary, when the amplitude is in a relatively high level, a dendrite grows slower under periodic pressure. As shown in Fig. 5, the length of a primary dendrite decreases with the increase of the amplitude of periodic pressure, and side branches are fewer under pressure with higher amplitude. When $\xi_{0}$ is 0.2 , there are no side branches on the small primary dendrite.

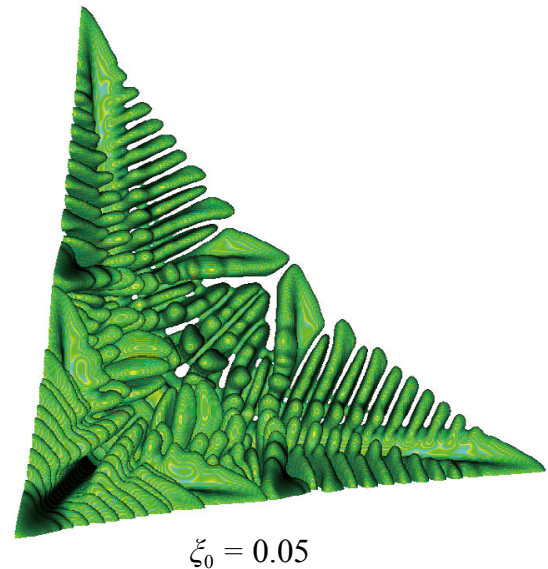

Fig. 5: Dendritic morphology at $t=30,000 d t$ under periodic pressure with different amplitudes
To reveal the underlying reason, tip velocity and total undercooling under periodic pressure with three amplitudes are shown in Fig. 6. It can be seen that total undercooling varies periodically in the three cases with different amplitudes. When $\xi_{0}$ is 0.1 and 0.2 , the valley of undercooling is too low for dendrites to grow. Especially when it is 0.2, the value of the lowest undercooling is even negative. The influence of low undercooling on dendrite growth can be found from the tip velocity curve. There is negative tip velocity in one period when $\xi_{0}=0.1$ and $\xi_{0}=0.2$. It can be deduced that dendrite (a)

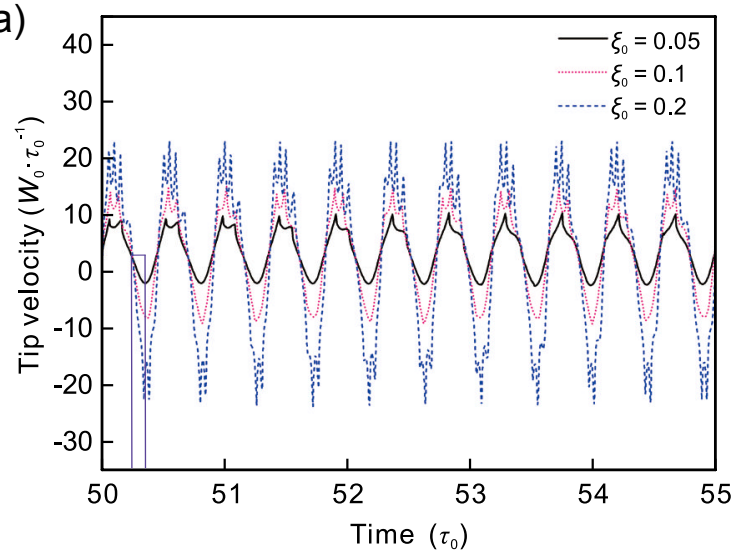

(b)

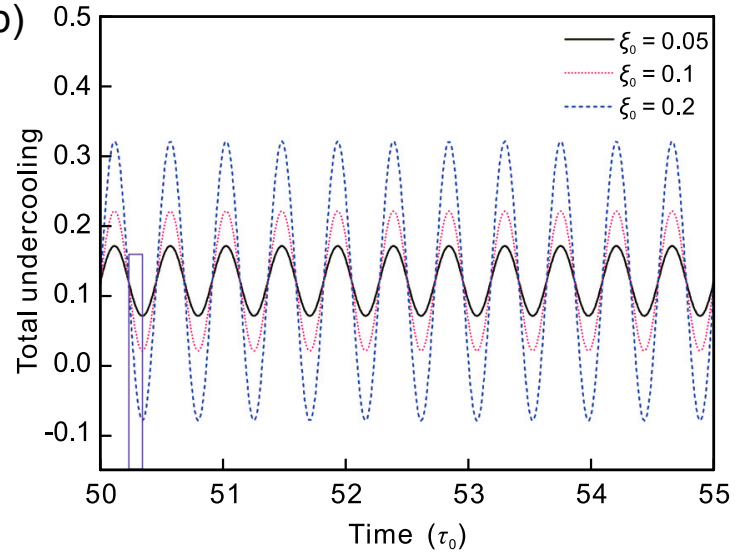

Fig. 6: Tip velocity (a) and total undercooling (b) solidified under periodic pressure with different amplitudes 
remelting occurs when applying periodic pressure in this study. Furthermore, as seen in Fig. 6, the lowest tip velocity decreases with the increasing $\xi_{0}$. That is, the highest dendrite remelting velocity increases with the amplitude of pressure. Higher remelting velocity may be the reason for a shorter dendrite under pressure with higher amplitude.

Similar to Fig. 3 and Fig. 4, the curves of total undercooling are symmetrical by the value of undercooling when $\xi=0$, and the curve of tip velocity when $\xi_{0}=0.05$ is also symmetrical by the value of velocity when $\xi=0$ as well. However, when the amplitude of periodic pressure is raised, the symmetry of tip velocity is gradually broken. The valley of tip velocity is lower than the symmetrical value of the peak. The differences of the highest tip growth velocity (positive tip velocity) and the highest tip remelting velocity (negative tip velocity) get greater under the periodic pressure with higher amplitude. Consequently, the average tip velocity decreases with the increasing amplitude of pressure, which is $3.4 W_{0} \cdot \tau_{0}^{-1}, 3.0 W_{0} \cdot \tau_{0}^{-1}$ and $2.2 W_{0} \cdot \tau_{0}^{-1}$ when $\xi_{0}$ is $0.05,0.1$ and 0.2 , respectively.

To further explore the asymmetrical phenomenon, the curves of tip velocity and total undercooling when $\xi_{0}=0.2$ in the area defined by the box in Fig. 6 are extracted, and the relationship of tip velocity and total undercooling is plotted and illustrated in Fig. 7. It is revealed that tip remelting velocity does not changes linearly with undercooling. The truth is, the accelerated velocity of tip remelting increases with the decline of undercooling. The greater the amplitude of periodic pressure, the faster the remelting velocity during one period. This is the underlying reason for the distinctions in average tip velocity and dendrite morphology when applied with periodic pressure with different amplitudes.

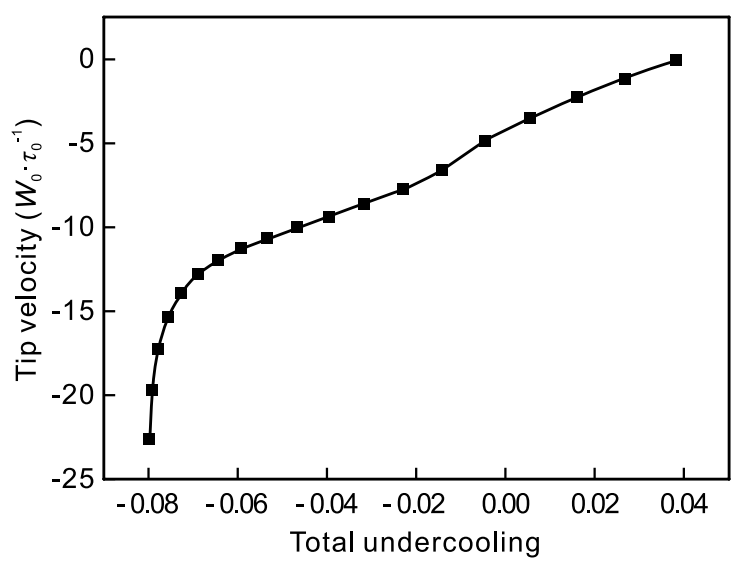

Fig. 7: Relationship of tip velocity and total undercooling when remelting occurs

\section{Conclusions}

The study compared the influence of atmosphere pressure, constant pressure which is higher than atmosphere pressure, and periodic pressure on dendritic growth and sidebranching behavior by using the 3 -D phase field method. Conclusions are drawn as follows:
(1) There are great distinctions in dendritic morphology and sidebranching behavior under the three kinds of pressure. Side branches are more luxuriant and with more developed high-order side branches when growing under higher constant pressure. Especially, when applied with periodic pressure, resonant sidebranching occurs, resulting in much more regular side branches, with the smallest SDAS in the three cases.

(2) The significant difference in dendritic morphology is determined by tip velocity which is modulated by total undercooling including pressure undercooling and temperature undercooling. In the case of constant pressure, tip velocity increases linearly with pressure, and in the case of periodic pressure, tip velocity varies in a periodic variation trend.

(3) A dendrite grows slower under periodic pressure with higher amplitude, which is caused by tip remelting due to low undercooling or even negative undercooling. It is revealed that the accelerated velocity of tip remelting increases with the decline of undercooling. The greater the amplitude of periodic pressure, the faster the remelting velocity during one period. This is the reason why average tip velocity decreases with the rise of amplitude of periodic pressure.

\section{Acknowledgements}

This work was supported by the National High Technology Research and Development Program of China (Grant No. 2018YFE0204300) and Institute Guo Qiang, Tsinghua University (Grant No. 2019GQG1010).

\section{References}

[1] Sawada T, Takemura K, Kitamura K, et al. Crystal growth by pressure control using a diamond anvil cell. Journal of Crystal Growth, 1988, 88(4): 535-536.

[2] Sawada T, Takemura K, Shigematsu K, et al. Dynamic pressure control for solution growth and its microgravity application. Journal of Crystal Growth, 1996, 158(3): 328-335.

[3] Sawada T, Takemura K, Shigematsu K, et al. Effects of gravity on a free dendrite of $\mathrm{NH}_{4} \mathrm{Cl}$ grown by dynamic pressure control. Journal of Crystal Growth, 1998, 191(1-2): 225-233.

[4] LaCombe J C, Koss M B, Tennenhouse L A, et al. The Clapeyron effect in succinonitrile: Applications to crystal growth. Journal of Crystal Growth, 1998, 194(1): 143-148.

[5] Kar P, LaCombe J C, Koss M B. Velocity and radius transients during pressure mediated dendritic growth of succinonitrile. Materials Science and Technology, 2004, 20(10): 1273-1280.

[6] Koss M B, LaCombe J C, Chait A, et al. Pressure-mediated effects on thermal dendrites. Journal of Crystal Growth, 2005, 279(1-2): 170-185.

[7] Yokoyama C, Tamura Y, Nishiyama Y. Crystal growth rates of tricaprin and trilaurin under high pressures. Journal of Crystal Growth, 1998, 191(4): 827-833.

[8] Sachdeva D, Tiwari S, Sundarraj S, et al. Microstructure and corrosion characterization of squeeze cast AM50 magnesium alloys. Metallurgical and Materials Transactions B, 2010, 41(6): 1375-1383.

[9] Masoumi M, Hu H. Influence of applied pressure on microstructure and tensile properties of squeeze cast magnesium Mg-Al-Ca alloy. Materials Science and Engineering: A, 2011, 528(10-11): 3589-3593. 
[10] Han Z Q, Pan H W, Li Y, et al. Study on pressurized solidification behavior and microstructure characteristics of squeeze casting magnesium alloy AZ91D. Metallurgical and Materials Transactions B, 2015, 46(1): 328-336.

[11] Huang X R, Han Z Q, Liu B C. Study on the effect of pressure on the equilibrium and stability of the solid-liquid interface in solidification of binary alloys. Science China Technological Sciences, 2011, 54(2): 479-483.

[12] Han G M, Han Z Q, Luo A A, et al. A phase field model for simulating the precipitation of multi-variant $\beta-\mathrm{Mg}_{17} \mathrm{Al}_{12}$ in Mg-Albased alloys. Scripta Materialia, 2013, 68(9): 691-694.

[13] Han Z Q, Zhu W, Liu B C. Thermomechanical modeling of solidification process of squeeze casting I. Mathematic model and solution methodology. Acta Metallurgica Sinica, 2009, 45(3): 356-362.

[14] Kim S G. A phase-field model with antitrapping current for multicomponent alloys with arbitrary thermodynamic properties. Acta Materialia, 2007, 55(13): 4391-4399.

[15] Kim S G, Kim W T, Suzuki T. Phase-field model for binary alloys. Physical Review E, 1999, 60(6): 7186-7197.

[16] Echebarria B, Folch R, Karma A, et al. Quantitative phasefield model of alloy solidification. Physical Review E, 2004, 70: 61604.

[17] Karma A. Phase-field formulation for quantitative modeling of alloy solidification. Physical Review Letters, 2001, 87(11): 115701.

[18] Eiken J, Böttger B, Steinbach I. Multiphase-field approach for multicomponent alloys with extrapolation scheme for numerical application. Physical Review E, 2006, 73(6): 066122.
[19] Pan H W, Han Z Q, Liu B C. Study on dendritic growth in pressurized solidification of Mg-Al alloy using phase field simulation. Journal of Materials Science \& Technology, 2016, 32: 68-75.

[20] Shang S, Guo Z P, Han Z Q. On the kinetics of dendritic sidebranching: A three dimensional phase field study. Journal of Applied Physics, 2016, 119(16): 164305.

[21] Shang S, Han Z Q, Sun W H, et al. A phase field model coupled with pressure-effect-embedded thermodynamic modeling for describing microstructure and microsegregation in pressurized solidification of a ternary magnesium alloy. Computational Materials Science, 2017, 136: 264-270.

[22] Börzsönyi T, Tóth-Katona T, Buka Á, et al. Dendrites regularized by spatially homogeneous time-periodic forcing. Physical Review Letters, 1999, 83(14): 2853-2856.

[23] Börzsönyi T, Tóth-Katona T, Buka Á, et al. Regular dendritic patterns induced by nonlocal time-periodic forcing. Physical Review E, 2000, 62(6): 7817-7827.

[24] Guo Z P, Xiong S M. On solving the 3-D phase field equations by employing a parallel-adaptive mesh refinement (Para-AMR) algorithm. Computer Physics Communications, 2015, 190: 89-97.

[25] Shang S, Guo Z P, Han Z Q, et al. Influence of periodic pressure on dendritic morphology and sidebranching. China Foundry, 2020, 17(4): 279-285. 